\title{
Presupuesto local y participación ciudadana en el ámbito europeo: una comparación entre las experiencias de Albacete y Salford
}

\author{
Carmen Pineda Nevot* \\ y Carlos Jesús Fernández Rodríguez**
}

Palabras clave: Participación ciudadana, proceso presupuestario, gestión local, desafección politica, transparencia.

\section{Introducción ${ }^{1}$}

En los últimos años, el Presupuesto Participativo se ha convertido en un tema de debate central en el ámbito de la políitca local, tanto en España como en otros países de la Unión Europea. Las razones para ello son variadas. Por una parte, el reconocimiento, a nivel internacional, de los modelos instaurados por el Partido de los Trabajadores brasileño en ciudades como Porto Alegre. El Orçamento Participativo (véase Abers 1998; Avritzer 1999; Baierle 2003) es una de las manifestaciones más visibles de un intento por hacer una «nueva política», alejada de la tecnocracia, más social y con mayor participación de los ciudadanos. Por otra parte, el descenso generalizado, en el caso europeo, de los niveles de participación electoral, fenómeno achacable a la falta de interés de los ciudadanos por una forma de hacer políica alejada de sus deseos. La participación directa de la ciudadanía en la gestión económica puede suponer una recuperación de su interés por los asuntos públicos, al permitir este sistema no sólo una mayor implicación en la toma de decisiones, sino también favorecer la legitimidad de la actuación (mayor respaldo social). Además, ofrece una vía de solución a las dificultades presupuestarias de los ayunta-

* Consultora de Administraciones públicas.

** Investigador del Departamento de Sociología de la Universidad Autónoma de Madrid.

${ }^{1}$ Este artículo fue presentado originalmente como comunicación en el VII Congreso de Ciencia Política «Democracia y Buen Gobierno”, organizado por AECPA. Quisiéramos agradecer la ayuda desinteresada prestada por Kezia Lavan (de Community Pride, en Salford), Carolyn Haslam (del Ayuntamiento de Salford), Carmen Campos (del Ayuntamiento de Albacete) y Javier Avilés (Coordinador del Foro de Participación de Albacete), y las sugerencias realizadas por Miguel Beltrán Villalva. 
tos, al identificar mejor las situaciones concretas y permitir el aumento de la eficacia y la eficiencia en términos de políticas de gestión (véase Pineda y Sanz, 1999; Pineda, 2002a, 2002b y 2004a).

No obstante, hay que tener en cuenta que la mayoría de las experiencias de participación presupuestaria puestas en marcha en Europa tienen poco que ver con la experiencia de Porto Alegre y con muchas experiencias similares de América Latina (Allegretti y Herzberg 2004). El contexto social, económico y político es muy distinto, y la dinámica de la democracia participativa también (Blanco, 2003). Además, el fenómeno es muy heterogéneo. Los países europeos tienen entre ellos bastantes diferencias en términos de cultura política, de competencia de los gobiernos locales, de política presupuestaria (Pineda 2004b). Lo que se llama presupuesto participativo no es exactamente lo mismo en Francia, Italia, Inglaterra, Alemania o España. No existe una metodología que pueda denominarse «presupuesto participativo», ni tampoco unas metodologías que puedan ser consideradas como un conjunto de variantes alrededor de una base común como ocurre en las experiencias latino-americanas, donde el modelo de Porto Alegre ha sido importado, adaptado y combinado en centenares de ciudades. Incluso dentro de cada país se pueden encontrar variaciones en su puesta en marcha, aunque los intercambios y las discusiones tienden a favorecer cierta homogeneización. Por eso los presupuestos participativos locales no constituyen en Europa una metodología, sino más bien un tema político y un movimiento de institucionalización de la participación ciudadana alrededor del presupuesto público (Sintomer 2004: 1).

En este artículo comenzaremos por tratar de definir, en primer lugar, qué es el presupuesto participativo; en segundo lugar, pasaremos a describir algunas de las diferencias existentes entre los sistemas políticos de los países donde se encuentran las experiencias que vamos a comparar; a continuación, señalaremos los rasgos más distintivos de la experiencia de Porto Alegre y de las experiencias española (Albacete) e inglesa (Salford); y, finalmente, realizaremos una comparación de las tres experiencias, haciendo especial referencia a las variables de diferenciación utilizadas en el análisis. El trabajo termina con un apartado de conclusiones.

\section{2. ¿Qué es el Presupuesto Participativo?}

Aunque en la mayoría de los textos que se refieren al tema no hay una definición explícita y sistemática, sí que existen aportaciones realizadas tanto por personas que trabajan directamente en el tema como por investigadores (Cabannes 2003a, 2003b). Unas son más técnicas, otras más políticas y otras tienen un alcance más general. Aunque no podemos recoger en este texto todas, sí que nos referiremos a cuatro que, nos parece, permitirán entender mejor los principios del presupuesto participativo.

La definición de Ubiratan de Souza ${ }^{2}$ (2001) del presupuesto participativo local es general, muy clara y sencilla. Para él, «el presupuesto participativo es un proceso de democracia directa, voluntaria y universal, en el que la población puede discutir y decidir sobre el presupuesto y las políticas públicas. De modo que el ciudadano no limita su participación a votar cada cuatro años, sino que también toma decisiones y controla la gestión del gobierno». Otra definición, más política que la anterior, es la de entender que se trata de una «esfera pública noestatal de cogestión». Acuñada por Tarso Genro3 ${ }^{3}$ (1997), alcalde de Porto Alegre durante los años 1993-1996 y 2000-2003, ha sido muy utilizada por el Partido dos Trabalhadores (PT) para señalar que la práctica del presu-

\footnotetext{
${ }^{2}$ Economista que trabajó en el Presupuesto Participativo de Porto Alegre y que pasó posteriormente a trabajar en la puesta en marcha del Presupuesto Participativo en el Estado de Rio Grande do Sul.

${ }^{3}$ Tarso Genro, además de haber sido dos veces alcalde de Porto Alegre, se ha presentado a gobernador del Estado de Rio Grande do Sul y es ministro del gobierno del Presidente Lula.
} 
puesto participativo tiene un potencial de transformación radical de las relaciones entre la sociedad civil y el propio Estado, entendiendo éste en un sentido amplio.

En cambio, para Fedozzi ${ }^{4}$ (1997) esta última definición limita la comprensión de la complejidad real del funcionamiento del presupuesto participativo, ya que no plasma el hecho de que en él convivan e interaccionen diferentes tipos de instituciones ${ }^{5}$. La definición que él nos ofrece es menos política y más matizada, describiendo el presupuesto participativo como una «esfera pública activa de co-gestión del fondo público municipal» que combina formas de democracia directa (las asambleas) con formas de democracia participativa semidirecta (Consejo del Presupuesto Participativo y los Foros de Delegados). También destaca una importante característica del mismo que no aparece en ninguna de las definiciones anteriores: el hecho de que los criterios y normas que rigen la toma de decisiones sean objetivos, impersonales y universales y los procedimientos, previsibles y conocidos por todos los participantes.

Para Sousa ${ }^{6}$ (1998), finalmente, el presupuesto participativo local es una estructura y un proceso de participación basado en tres grandes principios y un conjunto de instituciones que funcionan como mecanismos o canales de participación popular. Los tres principios son los siguientes: todos los ciudadanos tienen derecho a participar; la participación está dirigida por una combinación de reglas de democracia directa y de democracia representativa, y se realiza a través de instituciones de funcionamiento regular, cuyo régimen interno está determinado por los participantes; y, finalmente, que los recursos son distribuidos de acuerdo a un método objetivo, basado en una combinación de criterios generales y de criterios técnicos. La estructura institucional básica está formada por tres tipos de instituciones: unidades administrativas del ejecutivo municipal encargadas de dirigir el debate presupuestario con los ciudadanos; organizaciones comunitarias dotadas de autonomía frente al gobierno municipal, y compuestas principalmente por organizaciones de base regional; e instituciones de participación comunitaria con funcionamiento regular.

Con todas estas definiciones creemos que se puede conocer algo mejor qué es el presupuesto participativo. En primer lugar, se trata de un elemento novedoso en el ámbito político municipal, que no sustituye sino que complementa a la democracia representativa tradicional, buscando ampliarla y ejercer una función de control. En segundo lugar, se trata de un proceso que está en constante construcción: su funcionamiento es autorregulado, es decir, las instituciones que la componen son las que dictan sus propias normas de funcionamiento. Un último aspecto a destacar es el hecho de que la participación ciudadana tiene diversos grados: es directa en las asambleas, y semidirecta a través de la elección a representantes para el Consejo del Presupuesto Participativo y para los foros de delegados (Pineda 2004a).

Las experiencias de presupuesto participativo que vamos a comparar en este artículo se encuentran inmersas en contextos muy diferentes. Existen ciertas diferencias bien conocidas ${ }^{7}$ que van a ser determinantes en la configuración de las experiencias.

${ }^{4}$ Luciano Fedozzi fue responsable de una de las instancias de gobierno encargadas del presupuesto participativo en el primer gobierno del PT (1989-1992), investigador en la Universidad de Rio Grande do Sul y ha publicado dos libros y varios artículos sobre la experiencia de Porto Alegre (véase Fedozzi 1997 y 2000).

5 Según Fedozzi (1997 y 2000), no se debe considerar «esfera del presupuesto participativo» sólo a las instituciones creadas para gestionarlo (Consejo del Presupuesto Participativo y Foros de Delegados), sino que también forman parte del mismo las formas de organización de la población, que son autónomas con respecto a las esferas de gobierno, y aquellas estructuras del gobierno que tienen la función de organizar su funcionamiento.

${ }^{6}$ Boaventura de Sousa Santos, investigador y profesor de la Universidad de Coimbra, ha publicado diversos artículos y libros sobre el presupuesto participativo (véase 1998 y 2002).

${ }^{7}$ Existen numerosas distinciones entre ambos sistemas que no podemos desarrollar aquí en profundidad. A modo de ejemplo, podemos señalar que España cuenta con un texto constitucional único, lo que afecta a la capacidad de reformar las administraciones que además están suje- 


\section{Experiencias de Presupuesto Participativo}

\subsection{Porto Alegre}

La metodología de Porto Alegre ${ }^{8}$ (1.300.000 habitantes) ha sido hasta ahora la más influyente, y lo seguirá siendo en un futuro previsible. Aunque con el desarrollo de las experiencias y los intercambios crecientes la tendencia no está en la homogeneización, sino en el desarrollo de modelos mixtos -que, según los objetivos y las culturas políticas, recurren a varias metodologías para intentar combinar las ventajas y corregir los defectos de cada una-, la experiencia de Porto Alegre seguirá siendo, probablemente, la referencia central, porque es la referencia política más conocida y popular (ver Barceló y Pimentel 2002; Navarro 2000 y 2002; Utzig 1999). El Presupuesto Participativo se implantó en Porto Alegre en 1989, un año después del triunfo del Partido de los Trabajadores en las elecciones locales de 1988. La primera tarea de la nueva administración fue una reforma financiera, a la que siguió la organización de una esfera pública no estatal de poder que permitiera una administración compartida de la ciudad entre el sector público y la sociedad civil.

Sus características principales son las siguientes (Sousa 2002; Gret y Sintomer 2003):

- Se fundamenta sobre dos dimensiones, una territorial (con las asambleas de barrio o de sector) y otra temática ${ }^{10}$ (con asambleas que discuten sobre varias políticas públicas).

- Se basa en la participación abierta a todos los ciudadanos ${ }^{11}$, sin ningún estatus especial atribuido a las organizaciones comunitarias.

- Articula el nivel micro local (barrio o aldea) y el de ciudad.

- Articula asambleas abiertas a todos los ciudadanos y consejos de delegados ${ }^{12}$ que constituyen la cumbre del proceso y lo monitorizan (es, por tanto, una combinación de democracia directa y representativa) ${ }^{13}$.

tas a un derecho especial, el administrativo; mientras, en el Reino Unido las leyes y convenciones de equivalencia constitucional permiten a una mayoría del parlamento dictaminar una simple ley para reformar las Administraciones públicas, afectadas por el derecho común. Esto indica que, frente a una clara distinción entre Estado y sociedad en Europa, en el Reino Unido esos límites son más difusos, pues un ente público es igual que una empresa privada ante la justicia. Asimismo, frente al corporativismo dominante en el continente, en el ámbito británico existe un mayor pluralismo representativo. Existen además diferencias importantes en el sistema electoral, el modelo de gobierno local o la distribución territorial del poder. Sobre estas cuestiones clásicas en los estudios de ciencia de la administración, aconsejamos la consulta de trabajos como los de Chandler (2000), Olmeda y Parrado (2000), Ballart y Ramió (2000), Parrado (2002), Rodríguez Álvarez (2004) y Ziller (2005).

8 Porto Alegre es una ciudad de gran tradición democrática, con una sociedad civil fuerte y organizada. La oposición a la dictadura brasileña fue dirigida por los intelectuales, los sindicatos y el único partido de oposición legalizado, el Movimento Democrático Brasileiro (MDB). Dado que dicha oposición a nivel nacional no era posible, las organizaciones centraron su actividad en el fortalecimiento de los sindicatos y de los movimientos comunitarios, como las asociaciones de vecinos, los clubs de fútbol, los grupos culturales, etc. A principios de los ochenta, estos movimientos populares de base, hasta entonces muy heterogéneos en términos políticos y de organización, ganaron una nueva influencia en la política local. En 1983 se fundó la UAMPA (Unión de Asociaciones de Vecinos de Porto Alegre), que en 1985 realizó su primer congreso. En él, además de exigencias específicas (enseñanza, educación, empleo, etc.), pidieron la «efectiva democratización de las estructuras políticas en el nivel federal, estadual y local». Según Tarso Genro, cuando el Partido de los Trabajadores (PT) ganó por primera vez la alcaldía de Porto Alegre a finales de 1988 , existían cerca de mil organizaciones comunitarias en la ciudad.

${ }^{9}$ En 1989, el primer gobierno del PT comenzó dividiendo la ciudad en cinco regiones. No obstante, los dirigentes de las organizaciones comunitarias consideraron que éstas eran demasiado grandes, provocaban problemas de transporte y carecían de tradición política. Por eso se decidió, en colaboración con dichas organizaciones, dividir la ciudad en dieciséis regiones, división se ha mantenido hasta hoy (aunque se han ido introduciendo algunos pequeños cambios). En los últimos años se ha planteado la división de la región Centro, ya que al ser la que más población tiene (y ser la más rica) parece que sale favorecida con el sistema vigente.

10 A partir de 1994, empezaron a celebrarse asambleas por temas, utilizando la misma metodología que en las asambleas regionales.

${ }^{11}$ La participación de los ciudadanos en el proceso ha ido aumentando, con algunas pequeñas oscilaciones, conforme iba consolidándose el presupuesto participativo. Según la estimación del Gabinete del Alcalde, si se tienen en cuenta el centenar de reuniones intermedias regionales y temáticas, la participación total en el año 2000 fue, aproximadamente, de 100.000 personas (el 8\% de la población de la ciudad); pero si tenemos en cuenta los datos de participación ofrecidos por otras fuentes, los participantes sólo representan el 1,5\% de la población.

12 El criterio para determinar el número de delegados se ha modificado con los años. Inicialmente era un delegado por cada cinco personas que asistían a la asamblea; a comienzos de los noventa, el sistema cambió y se elegía un delegado por cada diez personas, después uno por cada 
- Utiliza criterios formales de repartición de los recursos dentro del marco de un reglamento explícito, que puede ser modificado por los participantes ${ }^{14}$; es decir, compatibilizando las decisiones y las reglas establecidas por los participantes con las exigencias técnicas y legales de la acción gubernamental (respetadas también las limitaciones financieras).

- Necesita un ciclo organizado de discusiones y toma de decisiones durante todo el año ${ }^{15}$.

- Permite discutir principalmente sobre el presupuesto de inversión, y especialmente sobre las obras ${ }^{16}$.

El cambio del equipo de gobierno de la ciudad -el PT perdió las últimas elecciones municipales- puede provocar algunos cambios en la metodología de esta experiencia. La nueva administración ya ha anunciado un nuevo modelo de gestión pública: la Gobernanza Solidaria Local (GSL), que consiste en la creación, en cada una de las dieciséis regiones en las que se encuentra dividida la ciudad, de un espacio llamado Gobernanza Local. Según César Busatto (CIDADE 2005), Secretario de Coordinación Política y Gobernanza Local del Ayuntamiento de Porto Alegre, «la Gobernanza Local es un forum ejecutivo, no deliberativo; es una articulación en red que busca crear pactos de co-responsabilidad. En este espacio no hay debate, ni votación, ni delegados». Este espacio está formado por los Consejos Municipales y los Forum Locales, la Cámara de Vareadores, el Tercer Sector, las Universidades, el Ministerio Público, el Poder Judicial, el Tribunal de Cuentas del Estado, el Poder Ejecutivo del Estado y Federal, el Comité Gestor del Gobierno Municipal, la Iniciativa Privada y el Presupuesto Participativo. Estos actores se repartirán la responsabilidad de concretar las demandas de la población con el poder público.

veinte y, posteriormente, un criterio más complejo. A propuesta del Coordinador de Relaciones con la Comunidad (CRC), este último criterio (excesivamente riguroso) fue abolido en 1997; en 1998-1999 se utilizó otro. A partir del 2000 se volvió a utilizar un número fijo: un delegado por cada diez participantes.

${ }^{13}$ La estructura institucional básica del Presupuesto Participativo está formada por tres tipos de instituciones. a) Unidades administrativas del Ejecutivo Municipal encargadas de dirigir el debate presupuestario con los ciudadanos: son el Gabinete de Planificación (Gaplan); el Coordinador de las Relaciones con las Comunidades (CRC); el Forum de las Asesorías de Planificación (Asseplas); el Forum de las Asesorías Comunitarias (Fascom); y los Coordinadores Regionales y Temáticos del PP (CROPs y CTs). Las dos más importantes son el CRC y el Gaplan. b) Organizaciones comunitarias dotadas de autonomía frente al gobierno municipal, y compuestas principalmente por organizaciones de base regional: consejos populares, uniones de barrios, etc. c) Instituciones de participación comunitaria con funcionamiento regular: son el Consejo del Presupuesto Participativo (COP); las Asambleas Plenarias Regionales; el Forum Regional del Presupuesto; las Asambleas Plenarias Temáticas y el Forum Temático del Presupuesto.

${ }^{14}$ El Presupuesto Participativo es un proceso social y político muy dinámico y complejo que ha permitido, hasta ahora, la participación de los ciudadanos en decisiones que han producido efectos redistributivos entre la población, la mejora de los servicios públicos y el equilibrio de la situación financiera del municipio. En el dinamismo del proceso ha tenido mucho que ver la actitud de las élites políticas de la ciudad, que han sido capaces de enfrentarse siempre a los problemas de una manera abierta, permitiendo en todo momento que los ciudadanos modificaran aquellos aspectos del Presupuesto Participativo que consideraran necesarios. Un ejemplo reciente de este talante es el impulso dado en 2001 por el alcalde Tarso Genro a la creación de un Grupo de Trabajo para la Modernización del Presupuesto Participativo, que tenía como objetivo reflexionar sobre la experiencia del PP y presentar propuestas de revisión y reformulación.

15 En la primera reunión el ejecutivo municipal da cuenta del presupuesto del año anterior, con explicaciones sobre la situación de las obras públicas decididas anteriormente. Ello permite el control por parte de los ciudadanos. También presentan la estimación que han realizado de los recursos financieros disponibles para el año siguiente y de las inversiones a ejecutar. La comunidad elige, en ese momento, parte de los delegados que la representarán en el Forum de Delegados. Entre la primera y la segunda reunión se celebran las llamadas intermedias, en las que la población discute las propuestas y son elegidos delegados (cerca de mil son elegidos anualmente). Cada región escoge cuatro entre las trece prioridades temáticas (limpieza básica, pavimentación, vivienda, salud, educación, asistencia social, transporte y circulación, áreas de ocio, deporte y ocio, iluminación pública, desarrollo económico, cultura y limpieza ambiental) y atribuye notas a sus prioridades. En la segunda reunión, los participantes en las asambleas regionales y temáticas eligen dos consejeros para el Consejo del Presupuesto Participativo (COP). Cuando finaliza dicha reunión en todas las regiones y temáticas, el Consejo está formalmente formado y se convierte en el órgano más importante para la definición del próximo presupuesto. En 2004 se aprobaron algunos cambios (los debates y la definición de prioridades comenzarán en abril o mayo, y la Asamblea Municipal se celebrará en julio) con el fin de simplificar el ciclo, lo que en su opinión favorecerá la ampliación del debate comunitario.

${ }^{16}$ Las inversiones son distribuidas de acuerdo con criterios que tienen en cuenta las diferencias entre las regiones. Actualmente (han cambiado con los años) los criterios son: a) Prioridad de la micro-región o comunidad (el peso de las prioridades es el siguiente: cuarta: 1; tercera: 2; segunda: 3 y primera, 4); b) Carencia del servicio o infraestructura (el peso de las prioridades es el siguiente: de 0,01\% a 14,99\%: 1; de 15\% a 50,99\%: 2; de $51 \%$ a 75,99\%, 3; y del 76\% en adelante, 4); y c) Población total de la región (el peso de las prioridades es el siguiente: hasta 25.000 habitantes: 1; de 25.001 à 45.000 habitantes: 2; de 45.001 à 90.000 habitantes: 3 y; por encima de 90.001 habitantes: 4). 
¿Cuál será entonces el papel del Presupuesto Participativo, que no es un actor y sí un espacio de deliberación y debate de la ciudad? Aunque no es posible saberlo todavía, sí que parece que, con estos cambios, se puede producir una disminución del poder de deliberación de las Asambleas del Presupuesto Participativo, de los Forum de Delegados y de las reuniones del Consejo del Presupuesto Participativo. De todas formas, habrá que esperar un tiempo para ver cómo funciona este nuevo modelo de gestión, y cómo reaccionan los vecinos si el nuevo equipo de gobierno pretende reducir sus espacios de deliberación y debate.

\subsection{Albacete}

Albacete (153.636 habitantes) ${ }^{17}$ comienza en el año 2000 a celebrar los Plenos de la Participación ${ }^{18}$, cuya filosofía es producto de la voluntad del nuevo equipo de gobierno ${ }^{19}$ de ir creando cauces de implicación de los ciudadanos en el desarrollo de la política municipal, para construir la ciudad entre todos. Este proceso, aunque ya iniciado, no está todavía concluido, y cada año se van incorporando nuevas aportaciones ${ }^{20}$, estudiando y modificando ${ }^{21}$ aquellos procedimientos que puedan no dar resultado (Avilés 2004).

A partir de las propuestas presentadas para el presupuesto municipal del año 2003 (más de dos mil), y después de un difícil trabajo de priorización, el Consejo del Presupuesto presentó las que consideraba líneas estratégicas de la ciudad, que debían impulsar el trabajo del año siguiente. Éstas eran: igualdad de oportunidades, sobre todo en la línea de conciliar la vida laboral y la familiar, para que sea posible la plena incorporación de la mujer al mercado laboral; integración social, con la atención a los colectivos que se ven más dificultados en su acceso a los recursos municipales, como es el caso de discapacitados, inmigrantes o presos; gestión más cercana, operativa y corresponsable de los recursos municipales; y la apuesta por un desarrollo sostenible, empezando por supervisar el gasto energético y aprovechar mejor nuestro medio.

Los vecinos ${ }^{22}$ pueden presentar propuestas ${ }^{23}$ sobre proyectos y servicios a través de una asamblea anual abierta a todos los ciudadanos. El Consejo del Foro, integrado por representantes de asociaciones de diversos ámbitos (vecinos, cultura, educación, ecología, inmigrantes, etc.) y ciudadanos no organizados, estudia las propuestas y después negocia con el ayuntamiento los proyectos que se realizarán, teniendo en cuenta los recursos del presupuesto y la viabilidad técnica y legal.

17 Todos los datos de población son del Padrón municipal de 2001.

18 Desde entonces se han celebrado tres plenos: el primero el 15 de febrero de 2000 en el que participaron 65 de las 323 asociaciones invitadas (el 20\%); el segundo el 15 de febrero de 2001 en el que participaron 83 de las 364 asociaciones invitadas (el 23\%) y; el tercero el 24 de enero de 2002 en el que participaron 277 de las 365 asociaciones invitadas (el 76\%).

19 Tras la pérdida de las elecciones municipales en 1995, la Agrupación Municipal del PSOE con su secretario general, Manuel Pérez Castell, elaboró un programa basado en la máxima cercanía posible con los vecinos, que se plasmó en lo que llamaron «Concejos Abiertos». Fue el origen de los Plenos de la Participación, que posteriormente se convirtieron en Foros de Participación.

20 Para supervisar el grado de ejecución de cada Pleno, a partir del II Pleno se creó la Comisión de Seguimiento, que se reúne de forma periódica y que está formada por los representantes de los colectivos que intervienen en cada Pleno, miembros del equipo de gobierno municipal y técnicos del Ayuntamiento.

${ }^{21}$ El IV Pleno de la Participación supuso un cambio en relación al III, al crearse el llamado Foro de la Participación. La misión de este foro era la elección en la asamblea de una mesa integrada por un representante de cada uno de los distintos colectivos que habían participado en anteriores plenos de la participación, así como la elección de un coordinador cuyo perfil debía ser el de una persona no identificada con ningún tipo de organización ni partido, y con proyección ciudadana. Posteriormente se eligió un consejo (compuesto por 27 personas) en el que están presentes representantes de los diferentes sectores, agrupados por la temática de su trabajo habitual. A partir de la aprobación, por parte del Foro del Reglamento, del Foro de Participación, pueden también formar parte del Consejo ciudadanos no organizados.

22 Hasta la aprobación del Reglamento del Foro de Participación el 17 de diciembre del 2003 sólo podían presentar propuestas las asociaciones. A partir de esa fecha lo pueden hacer también los ciudadanos no organizados.

${ }^{23} \mathrm{Al}$ presupuesto de 2005 se presentaron en la Asamblea del Foro de la Participación 525 propuestas. 
Posteriormente, hace un seguimiento de la ejecución de los proyectos aprobados. Para la clasificación de las propuestas, se han elaborado y aprobado este último año unos criterios de priorización ${ }^{24}$ que tienen en cuenta cuestiones como el grado de necesidad (población destinataria), la población beneficiada (número de habitantes), la carencia del recurso o inexistencia de la prestación del servicio en relación a otros barrios de la ciudad, y el número de ciudadanos que hacen la petición del recurso o servicio. El proceso de Albacete supera ya el simple debate sobre proyectos de inversión, al discutirse otros gastos e ingresos (tasas y precios públicos). El Foro ha asumido también un papel de mediador social entre la ciudadanía y el Gobierno Local (en el «conflicto del botellón», la subida del precio en las tarifas del agua, el servicio de autobuses, etc.). Además, ha realizado un análisis sobre la infraestructura urbana que permite especificar las demandas de las asociaciones en el proceso y hacer un seguimiento de éstas (véase Pineda 2003; Pérez Castell 2004).

Las dificultades ${ }^{25}$ encontradas para que converjan, de una manera efectiva, los tres ejes que sostienen el pro$\operatorname{ceso}^{26}$-el eje técnico, que comprende las áreas del ayuntamiento; el eje político y finalmente el Foro de la Participación-, junto con la falta de encaje entre los tiempos de presentación de propuestas y los plazos que marca la ley para la elaboración de los presupuestos municipales, han llevado al Consejo del Foro de la Participación a tomar la decisión de que, para el presupuesto del 2006, no se presenten propuestas, y se aproveche este año para adaptar el calendario del Foro ${ }^{27}$ a los plazos del presupuesto municipal. Para los presupuestos del año 2007, no sólo pretenden respetar los plazos de presentación de propuestas, sino también cuantificar el número de las mismas para conocer mejor cuánto vale lo que se pide. Con ello, el proceso ganará en credibilidad (Pineda 2004c).

\subsection{Salford}

Salford ${ }^{28}$ (216.103 habitantes), aunque ciudad con ayuntamiento propio ${ }^{29}$ (Salford City Council), pertenece al área metropolitana conocida como Greater Manchester, esto es, una serie de ciudades y pueblos limítrofes con Manchester, en el norte de Inglaterra (que comprende, además de Manchester, los municipios de Salford, Bolton, Bury, Oldham, Rochdale, Stockport, Tameside, Trafford y Wigan, sumando entre todos ellos una población de 2.482.328 habitantes). La ciudad está, de este modo, muy cerca de una de las conurbaciones más importantes de Inglaterra y a menos de dos horas en coche de un área donde se estima vive el 60\% de la población de Inglaterra (grandes ciudades como Liverpool, Sheffield, Leeds, Bradford o Newcastle). No está claro que el proceso que se

24 Para el presupuesto del 2005, una comisión del Consejo elaboró unos criterios para la priorización de las propuestas, que se presentaron y aprobaron en el último Foro de la Participación.

25 Otras dificultades con las que se han encontrado en estos años son las dificultades de comunicación y los tiempos de funcionamiento. En lo que respecta a la comunicación, no deja de ser un problema que la experiencia sea más conocida y reconocida en toda España y Europa que en la propia ciudad de Albacete; en cuanto a los tiempos, el Foro ha funcionado muchas veces demasiado rápido, sin darse un tiempo para reflexionar sobre aspectos teóricos fundamentales, y en otros casos, demasiado lento para las expectativas de los ciudadanos. Estas y otras cuestiones necesitan un periodo de reflexión.

${ }^{26}$ En el reglamento del Foro se arbitró un canal para que estos tres ejes convergieran de una manera más efectiva. Fue lo que se denominó la oficina del presupuesto participativo, una mesa técnica. Sin embargo, ésta no se ha puesto todavía en marcha.

${ }^{27}$ A partir del 2006 las propuestas se recogerán de enero a mayo, y en mayo-junio se presentará la propuesta de presupuesto participativo y se realizará la asamblea.

${ }^{28}$ Los orígenes de Salford se remontan al siglo XIII, si bien la ciudad que hoy en día conocemos tiene sus orígenes en la revolución industrial británica, con la construcción de varios canales (Bridgewater Canal, Manchester Ship Canal) y el ferrocarril. Ciudad industrial muy próxima a Manchester, creció notablemente hasta después de la Segunda Guerra Mundial, desarrollando una economía muy próspera basada en el transporte (a través de los puertos de los canales), ingeniería pesada, industrias textiles y químicas y minería (carbón especialmente). Con la crisis industrial, la ciudad entró en declive (al igual que Liverpool, Leeds, Bradford y otras) y en la actualidad se encuentra en plena fase de post-crisis, con variados problemas sociales.

29 El Ayuntamiento de Salford cuenta con 60 concejales o councillors que sirven por un máximo de cuatro años y son responsables de los veinte distritos electorales. Es una circunscripción que, tradicionalmente, ha votado al partido laborista: se puede considerar un feudo histórico de la izquierda británica. Del área de Salford sale además elegido un Member of Parliament que va al Parlamento Británico. 
está desarrollando actualmente en Salford sea de puro presupuesto participativo, si bien el ayuntamiento lo considera como tal: no obstante, sí está claro que se trata de una serie de experiencias cuya meta final es la de devolver una parte de las decisiones en materia de gasto e inversión pública a la ciudadanía. Por tanto, depende de qué entendamos por presupuesto participativo: un foro donde la gente da su opinión, o un proceso en el que la ciudadanía toma decisiones directas en materia presupuestaria. La realidad es que no existe una parte del presupuesto que sea decidida por completo por la ciudadanía, sino más bien un compromiso futuro y, sobre todo, un importante esfuerzo informativo. A través de esta iniciativa de Participatory Budgeting (PB), se pretende conocer los puntos de vista de los residentes en el municipio sobre las prioridades de gasto. Por tanto, no es todavía presupuesto participativo pero están en el camino hacia el mismo: así, Salford cuenta con un proceso de consulta presupuestaria y una serie de iniciativas adicionales que explicaremos a continuación.

El Ayuntamiento debe presentar, anualmente, un presupuesto para hacer frente al coste de proporcionar un amplio abanico de servicios públicos, y que marcan el nivel del impuesto local (Council Tax) para el año ${ }^{30}$. El proceso de consulta presupuestaria se denomina Budget Consultation Process, y comenzó hace siete años por iniciativa del gobierno local laborista. Cuenta con el importante apoyo de la ONG Community Pride. El Budget Consultation Process dura tres meses, desde noviembre hasta enero. Se consulta la visión del ciudadano de los Presupuestos, la cantidad y calidad de los mismos, opciones para incrementar y reducir el Presupuesto, etc. Los resultados de estas consultas se envían a los principales responsables y, a partir de ahí, se elabora un documento con dichas cuestiones (que se transmite al Ayuntamiento). Las formas de participar en la consulta son a través de consultation meetings que se realizan cada cierto tiempo (al comienzo del proceso en el Ayuntamiento de Salford, actualmente en locales en diferentes barrios), o con formularios que se pueden enviar a través del correo electrónico o por vía postal. Hay una política definida por parte de los poderes locales de nuevas formas de e-government. Así, hay dos formas de participar dando una opinión vía on-line: por un lado, se facilita a los ciudadanos dar su opinión (Have your say) en un foro; por otro, se lleva a cabo un cuestionario con preguntas relativas a prioridades de gasto y aspectos en los que se podría mejorar la eficiencia. Los resultados de estas consultas se presentan en la página web del ayuntamiento: <http://www.salford.gov.uk>

Junto a este proceso de consulta, tanto el Ayuntamiento de Salford como Community Pride se han involucrado en dar a conocer el funcionamiento del proceso presupuestario, de modo que los ciudadanos conozcan cómo pueden intervenir en el mismo. Este sería un paso fundamental, ya que mejoraría el conocimiento técnico de los implicados en el proceso, aumentaría su autonomía (capacity building) y promovería, a nivel político, la puesta en práctica del presupuesto participativo. Incluso han invitado a los responsables del presupuesto participativo de Porto Alegre para informarse mejor acerca de lo que se está haciendo en Brasil (Wainwright 2000).

Se podría afirmar, por tanto, que el proceso se encuentra todavía en una fase más «informativa» que de toma de decisiones. No obstante, durante el ejercicio del 2004 se procedió a «devolven $\rangle^{31}$ una pequeña parte del presupuesto a nueve de los llamados Community Comittees. Estos comités toman a su vez las decisiones a través de

${ }^{30}$ El presupuesto global con el que cuenta el gobierno local de Salford procede de tres grandes partidas. Más de un $53 \%$ son ayudas presupuestarias del gobierno central, un $21 \%$ procede de impuestos sobre sociedades no distribuidos y, finalmente, un $25 \%$ se financia con impuestos locales (unos 70 millones de libras). Los servicios que financian los impuestos locales son los facilitados por el Ayuntamiento de Salford y algunos pertenecientes a lo que se conoce como el área del Greater Manchester, concretamente policía y bomberos. La cantidad que se paga en los impuestos locales depende de la banda en la que el hogar en el que se habita esté incluido. De este modo, el 55\% de los habitantes de Salford viven en casas pequeñas que entran dentro de la categoría A, pagando 856,84 libras esterlinas anuales. Si tomamos una casa de la categoría D, los gastos ascenderían a 1285,26 libras anuales, de las que el 89\% financiarían servicios del Ayuntamiento de Salford, el 7,7\% financiarían la policía (Greater Manchester Police Authority) y un 3,3\% el servicio de bomberos (Greater Manchester Fire \& Civil Defence Authority).

31 Utilizamos la expresión «devolver» en el sentido de devolution, expresión británica que denomina el proceso por el que ciertas decisiones políticas son cedidas por la Administración a los ciudadanos. 
los llamados Sub Budget Groups, formados por concejales (councillors), funcionarios, proveedores de servicios y ciudadanos. Estos comités pueden solicitar fondos a través de un programa de pequeñas ayudas. La información recogida durante el proceso de consulta es discutida en estos comités, y de ahí pasa al Ayuntamiento de Salford donde se discute cómo ejecutar el presupuesto. El responsable de este proceso (y de su puesta en práctica) es el Assistant Director de la división financiera del Ayuntamiento, que depende a su vez de la sección de Customer and Support Services.

La comunicación de estas iniciativas se realiza a través de varias medidas: información en la página web del ayuntamiento de Salford; comunicados de prensa; creación de una base de datos con los ciudadanos interesados en el proyecto; copias del presupuesto y las decisiones para los asistentes a diferentes reuniones; y, para el año 2006, creación de un citizen's panel.

\section{Variables de diferenciación}

Al estudiar las experiencias de participación presupuestaria local existentes tanto en España como en otros países vemos que presentan diferencias entre ellas (Pineda 2004b). Para facilitar la comprensión de éstas, hemos elegido dos variables de diferenciación ${ }^{32}$ : objetivos y actores. En primer lugar plantearemos las variables que vamos a utilizar, para luego observarlas en las experiencias que vamos a comparar.

\subsection{Objetivos}

Los objetivos son muy distintos pero se pueden diferenciar, a nivel analítico, tres tipos potenciales que resumen los varios objetivos concretos que explicitan los actores (Gret y Sintomer 2003; Bacqué, Rey y Sintomer 2004): un nivel administrativo, en el que se puede utilizar la participación para mejorar la gestión pública; un nivel social, lo que implica pensar que la participación presupuestaria puede influir sobre las relaciones sociales; y un nivel político, en el que parece posible convertir esta metodología innovadora en un instrumento para «democratizar la democracià, como dicen los brasileños de Porto Alegre.

\section{- Nivel administrativo.}

Este nivel es fundamental en Brasil, probablemente porque se pueden constatar efectos concretos en términos de lucha contra la corrupción, de reducción del clientelismo y de modernización de la gestión pública (Utzig 2000).

Los análisis realizados (Sintomer 2004) demuestran que la introducción de una experiencia de participación presupuestaria está casi siempre relacionado con un proceso de modernización administrativa, sea porque para éste se necesita el uso de un instrumento fuerte de participación, sea porque el desarrollo de la participación presupuestaria no puede seguir adelante sin trasformar la Administración. No obstante, esta relación no es automática, pues en países empeñados en modernizar su Estado como los de Europa del Norte o el Reino Unido el desarrollo del presupuesto participativo es inexistente, o casi.

32 Para comparar las experiencias existen muchas variables de diferenciación, pero en este caso se utilizan las de la Investigación Internacional Comparada sobre Democracia Participativa que se está realizando en el Centro Marc Bloch de Berlín, coordinado por el profesor Yves Sintomer con el apoyo de la Fundación Hans-Böckler y la Delegación de la Ciudad del Gobierno francés. 
Esta transformación puede realizarse de distintos modos. Uno de ellos es el de mejorar la gestión de proximidad, articulando la cooperación trasversal de los distintos servicios públicos en un territorio: ello permite un acceso más fácil a estos servicios, nombrando en cada distrito un responsable administrativo y un responsable político para seguir todos los problemas y solicitudes de la ciudadanía, y desarrollando la participación para conocer mejor las necesidades de la gente. Otro es más ambicioso, pues lo que pretende es un proceso de modernización general. En este caso la participación se concibe como una de las vías de la modernización administrativa: la idea tiene que ver con romper compartimentos estancos en las administraciones, ofrecer servicios más accesibles, tener más en cuenta las demandas de la gente, proponer objetivos de calidad, desarrollar un presupuesto por objetivos en vez del presupuesto clásico, y reformar la dirección interna de la administración. La fuerte crisis financiera de los municipios ha contribuido también a hacer del presupuesto participativo un instrumento potencial de consolidación de las finanzas, a través de la responsabilización cooperativa de todos los actores y de una mejor legitimación de las decisiones (Pires 2000a).

El caso de Salford está ligado a una estrategia financiera vinculada a la visión del equipo de gobierno del Ayuntamiento, que pretende hacer de Salford una ciudad que la gente elija para vivir y trabajar. Para ello intentan mejorar la calidad de vida de los ciudadanos, pretendiendo llevarlo a cabo a través de las siguientes promesas: la mejora de los servicios sanitarios; la reducción de la delincuencia, un objetivo absolutamente prioritario; la promoción del aprendizaje, el ocio y la creatividad; la inversión en la gente joven; la promoción de la inclusión social, luchando contra la pobreza y las desigualdades sociales; la creación de prosperidad y la mejora de la calidad de vida.

Se trata por tanto de un enfoque dirigido a la mejora de la calidad de los servicios prestados. Para llevar adelante estas premisas, se busca construir un presupuesto con el asesoramiento de distintas instituciones y entidades independientes. Para ello, cuentan con la asesoría del Gobierno, inspecciones, la encuesta de Calidad de Vida, objetivos designados por el Council's Local Public Service Agreement, y los mensajes de los ciudadanos que consultan el presupuesto. Existen, por supuesto, una serie de restricciones al presupuesto: la identificación de unos objetivos de gasto concretos, y de formas de ahorro que hagan el presupuesto más eficiente; la consideración de los efectos del recorte de ciertas prestaciones de servicios públicos; el mantenimiento de los impuestos locales a un nivel razonable; y la protección de la gente que depende de los servicios públicos, los cuales deben ofrecerse gratuitamente.

El caso de Albacete es distinto, porque aunque se encuentra inmerso en un proceso de modernización y descentralización ${ }^{33}$, no es ésta una de las prioridades de su experiencia.

\section{- Nivel social}

En Brasil el presupuesto participativo es un instrumento de las clases populares, un método privilegiado para invertir las prioridades a favor de los pobres y las mujeres (Sintomer 2005). No ocurre lo mismo en España, aunque eso no significa que no haya objetivos sociales sino que son simplemente distintos. Existen en las experiencias españolas temas de inclusión social y de carencias de servicios e infraestructuras, pero no hay mecanismos para la movilización de los más desfavorecidos. Tampoco se ha estudiado hasta ahora si la introducción de la participación presupuestaria puede realmente modificar las prioridades sociales, como ha ocurrido en el caso en Porto Alegre (véase Marquetti 2000 y 2003; Wampler 2002).

${ }^{33}$ En el mes de diciembre de 2004, la Asamblea Legislativa de la Comunidad de Castilla-La Mancha aprobó la incorporación de Albacete a la Ley 57/2003 de Medidas para la Modernización del Gobierno Local. 
El caso de Salford es, en este sentido, especial. La ciudad de Salford experimentó, entre 1971 y 1991, una notable pérdida de población, casi un $20 \%$ de hecho (unas 60.000 personas). Esta importante pérdida de población guarda una relación directa con la crisis de la industria, que afectó a esta ciudad al igual que a muchas otras del norte de Inglaterra. Entre 1965 y 1991, se estima que se perdieron alrededor de 49.000 empleos, esto es, el $32 \%$ del empleo existente en el municipio. Las principales pérdidas se registraron en el sector industrial y no pudieron ser compensadas por las inversiones en el sector servicios. Existen en la actualidad aproximadamente 6.500 empresas en Salford, de las que el 85\% son PYMES con menos de 25 empleados. La industria de la ciudad, por tanto, ha sufrido un declive muy importante, desarrollándose por el contrario empresas del sector servicios como banca, servicios financieros, etc. Durante la última década han aumentado notablemente los empleos a tiempo parcial, especialmente entre la población masculina (con una notable incidencia también entre los jóvenes varones). Se ha convertido, así, en una ciudad de contrastes: mientras zonas como Central Salford o Salford Quays se han convertido en zonas de negocios con cierta proyección, barrios como Ordsall, Weaste, Eccles, Pendleton, Broughton o Blackfriars sufren las consecuencias de la crisis, con problemas sociales diversos como delincuencia, casas viejas y mal acondicionadas, situaciones de pobreza y, sobre todo, una tasa de desempleo relativamente alta para Inglaterra, un 14,6\%. El paro castiga sobre todo a la población masculina y a los jóvenes, lo que ha favorecido la aparición de fenómenos de gamberrismo juvenil y numerosos robos.

El ayuntamiento de Salford persigue dejar atrás una cierta imagen de la ciudad como lugar problemático, y esto es evidente aunque no figure de una manera explícita en la publicidad de la iniciativa (sí es muy explícito este aspecto en los resultados de las consultas). Se observa además que las dos partidas presupuestarias más elevadas corresponden a los conceptos educación y servicios sociales. De este modo, a través de una profundización en la democracia, se persigue la construcción de una comunidad con más autonomía, que pueda empujar a Salford fuera de una cierta crisis social. Por otra parte, no es sorprendente que parte de los miembros de Community Pride procedan de asociaciones religiosas con experiencia en el combate contra la pobreza, además de contar con el apoyo de la ONG Oxfam, también dedicada a los mismos objetivos.

\section{- Nivel politico}

La reflexión sobre la democracia constituye uno de los puntos principales de la agenda política en el inicio del siglo XXI. Si el siglo anterior estuvo marcado por la afirmación democrática como elemento central de la política, el debate contemporáneo da un paso más y se preocupa de la calidad de estos regímenes. La necesidad de reflexionar sobre la calidad de las democracias contemporáneas se relaciona con la crisis del Estado, especialmente las crisis vividas por sus instituciones representativas. El debate sobre la reforma del Estado debe abarcar una amplia discusión sobre los instrumentos por medio de los cuáles la democracia debe discurrir. Se trata de la necesidad de buscar mayor legitimidad en los procesos democráticos, fomentando, con eso, gestiones públicas transparentes y mayor responsabilidad.

Entre los problemas más evidentes está la distancia entre representante y representado, el no cumplimiento de las promesas de campaña y la ausencia de mecanismos de control de los representantes por parte de los representados. El reconocimiento de esas dificultades y el debate sobre sus soluciones llevan al replanteamiento de la naturaleza y las estructuras de la representación política. Entre las formas alternativas que se plantean están las experiencias de democracia semidirecta o participativa: la democracia representativa puede combinarse con democracia participativa, lo que facilita una mayor presencia de los ciudadanos en el proceso de toma de decisiones. Los dos modos de ejercicio de poder no constituyen sistemas excluyentes, sino complementarios y compatibles ${ }^{34}$.

\footnotetext{
34 Recordemos que la Carta Europea de Autonomía Local garantiza expresamente la democracia directa y defiende su coexistencia con la democracia representativa.
} 
Esta complementariedad no significa, sin embargo, una ausencia de conflictos. El fortalecimiento de mecanismos de participación directa o semidirecta lleva a una nueva configuración de poder, y pone en evidencia las deficiencias del sistema representativo. La apertura de espacios de participación ciudadana provoca una tensión constante entre las dos legitimidades (la de los representantes y la de los representados), y es necesario encontrar un punto de equilibrio entre ambas.

Para los responsables de Porto Alegre el presupuesto participativo es un buen ejemplo a escala local de lo que sería, en realidad, un nuevo modelo de democracia; es decir, una nueva forma de relacionarse el Estado con los ciudadanos y con los distintos agentes existentes en la sociedad (Rodríguez Prieto 2005). Se trataría de uno de los elementos, tal vez el principal, de una respuesta a la crisis del Estado en su forma generada por la modernidad (Genro 2000). Para ello se ha creado un sistema que completa la democracia representativa con elementos de democracia directa. Ello implica la emergencia de un cuarto poder, el de los ciudadanos cuando toman las decisiones directamente o a través de delegados estrechamente controlados. Es un poder que no sustituye, sino que se articula con los tres poderes clásicos, el ejecutivo, el legislativo y el judicial (Bacqué, Rey y Sintomer 2004).

En cambio, en Europa, la mayoría de las experiencias de presupuesto participativo no son todavía deliberativas, sino consultivas, y las decisiones las toman al final los políticos. Ocurre siempre así en Francia, Alemania, Bélgica, Inglaterra y Portugal, por los menos cuando se trata de decisiones más allá del nivel de barrio. En España, la situación es un híbrido entre la de los otros países europeos y la de Porto Alegre.

En el caso de Salford, hay que destacar también que la participación en las últimas elecciones locales ha sido extremadamente baja, un 25\%. La pérdida de credibilidad de las instituciones democráticas ha sido esencial para que el Ayuntamiento se haya decidido a poner en marcha estos proyectos. Por otro lado, la ciudad tiene importantes problemas sociales, y una mayor implicación de los actores podría ser una medida de favoreciese la búsqueda de soluciones.

\subsection{Actores}

Existen dos diferencias muy importantes entre los presupuestos participativos locales europeos y la experiencia de Porto Alegre ${ }^{35}$. La primera es que, en Europa, son casi siempre procesos «desde arriba». La confluencia entre un movimiento desde abajo y uno desde arriba, que se dio en Porto Alegre, no suele tener lugar, con la excepción del caso de Albacete. En muchos países europeos, la crisis de los partidos tradicionales es fuerte, el prestigio de la política está mucho más bajo que antes. Desde los años setenta, muchos movimientos urbanos han reivindicado más participación. En este sentido, se puede decir que el sistema político empieza con los presupuestos participativos a integrar las críticas que habían sido hechas en este terreno. Sin embargo, en los últimos años, no se ha visto a los movimientos sociales reivindicar una participación en la definición del presupuesto municipal y obtener satisfacción. La iniciativa del proceso la toma siempre o casi siempre el alcalde y su equipo, y muchas veces tiene problemas para movilizar a la gente que tiene dudas sobre los efectos reales de la participación.

La segunda diferencia es que en Porto Alegre, y en casi todas las experiencias latino-americanas, son las clases populares los actores principales del proceso, este hecho provoca las críticas de una parte de las clases altas a quienes no les gusta este «instrumento de los pobres» (Pires 2000b). En España, en cambio, son las clases medias las que son más activas, siendo los jóvenes, los extranjeros o inmigrantes y los más pobres los que per-

35 En muchas ciudades brasileñas y latinoamericanas, los presupuestos participativos vienen también desde arriba (véase Avritzer 1999; y Avritzer y Navarro 2003). 
manecen ausentes o marginados en los presupuestos participativos. Si miramos a los actores más concretos, hay que destacar en España el papel fundamental de los partidos políticos. De hecho, son más bien dirigentes locales de los partidos los realmente activos, ya que no existe ningún país en que un partido haya aplicado experiencias de participación presupuestaria en todos los municipios en los cuales detenta el poder. También es necesario señalar que, aunque son los partidos de izquierda los que están más interesados en la temática, en España existen ya experiencias en municipios gobernados por partidos conservadores.

Las fundaciones y las ONG's ${ }^{36}$ juegan también, en muchos países, un papel importante en la difusión de la idea de presupuesto participativo, en la elaboración de las metodologías necesarias y en el asesoramiento técnico del proceso. En España, excepto en Cataluña (la Fundación Jaime Bofill), no han participado de ninguna manera. En Inglaterra, en cambio, la labor desarrollada por la asociación Community Pride Initiative en este proceso ha sido muy importante. Community Pride, creada en 1999, es una asociación formada por varios grupos asociados: el CAP (Church Action on Poverty), la diócesis de Salford, los metodistas, la United Reform Church e iglesias baptistas de Manchester, pero también otros activistas y asociaciones ciudadanas de todo tipo. Cuenta además con el apoyo de la ONG Oxfam. Ha sido el grupo que más ha contribuido a la movilización de la ciudadanía a través de voluntarios que han informado del proyecto. Para Community Pride, hay tres ingredientes que se hacen necesarios para el desarrollo del presupuesto participativo: voluntad política, compromiso por parte de la comunidad y un cierto conocimiento técnico en estas cuestiones (véase Community Pride 2003, 2005a y 2005b).

Un tercer tipo importante de actores son las asociaciones y los diversos colectivos de vecinos y de ciudadanos (Arenilla 2004). Muchas metodologías están concebidas para implicar a los ciudadanos que no están organizados y no son muy favorables a los colectivos. En muchas partes, esto ha generado tensiones entre el presupuesto participativo y las asociaciones, hasta provocar casi la parálisis del proceso, como ha ocurrido en Córdoba. En otros lugares, donde la metodología es más favorable a la participación de las asociaciones, éstas juegan un papel más dinámico y a veces hasta central, como en Albacete donde ocupan casi todo el espacio.

Asimismo, hay que referirse a la implicación de los funcionarios y técnicos, que no es homogénea (Villasante 2004). Las personas que se ocupan directamente del presupuesto participativo o que están encargadas de las relaciones con la ciudadanía juegan un papel muy activo y, de hecho, son las que hacen que el mecanismo pueda funcionar. No obstante, todavía la gran mayoría de los funcionarios tiene dificultades para incorporar la participación en su trabajo cotidiano, por varias razones: porque tienen miedo a perder su poder, porque piensan que la gente podría amenazar la racionalidad administrativa o porque, simplemente, no han sido formados para enfrentarse a esta nueva problemática. En el caso de Salford, este panorama es complejo debido al solapamiento entre funcionarios y personal de agencias ${ }^{37}$.

La última diferencia notable entre los presupuestos participativos europeos y el de Porto Alegre (y de los demás casos latinoamericanos) es el papel del consejo municipal. En América Latina, el legislativo local queda

\footnotetext{
36 Las Fundaciones han sido tal vez el actor principal en Alemania a nivel nacional, y una pequeña ONG llamada «Arbeitsgruppe Burgerhaushalt Berlín» ha sido muy activa en la capital del país. En Francia, dos ONG's nacionales, l'ADELS (que ofrece cursos de capacitación en temas de gestión local y publica la revista Territoires) y DRD (Democratizar Radicalmente la Democracia) han hecho un trabajo importante.

${ }^{37}$ Las Agencias se crearon, a partir de 1988, con el fin de mejorar la prestación de los servicios públicos. El establecimiento de las agencias británicas sigue explícitamente el modelo de la teoría de la agencia, por la que el agente debe cumplir lo estipulado en el contrato establecido con el principal. El contrato se plasma en un documento marco, y en él se estipulan las obligaciones de la agencia de conseguir los objetivos marcados. Ésta goza de notable autonomía en la gestión de sus recursos, y puede operar casi como una empresa privada. En algunos casos, las agencias se ven obligadas a competir con otras empresas para la prestación de un servicio determinado; incluso varias agencias compiten entre sí para obtener los recursos públicos, como ha ocurrido parcialmente en el ámbito universitario y en el sanitario. La independencia está, por tanto, condicionada por el documento marco, que funciona a modo de contrato.
} 
marginado en el proceso del presupuesto participativo, dado que se basa sobre todo en un diálogo entre el ejecutivo local y la sociedad civil. El sistema presidencialista que se aplica en casi todas las ciudades latinoamericanas, con la elección directa del alcalde, contribuye probablemente a esta situación. En cambio, en Europa, donde el sistema más común a nivel municipal es el sistema parlamentarista -en el cual el alcalde es elegido por el pleno municipal y comparte entonces el mismo color político-, el pleno municipal juega normalmente un papel mayor que en América Latina. Por supuesto, el impulso del alcalde (o de los tenientes alcaldes) es fundamental, y muchas veces es él quien toma las decisiones reales.

En el cuadro siguiente podemos ver las diferencias existentes entre las tres experiencias: la de Porto Alegre (Brasil), la de Albacete (España) y Salford (Reino Unido).

\section{CUADRO 1}

Diferencias entre las experiencias de Porto Alegre, Albacete y Salford

\begin{tabular}{|c|c|c|c|}
\hline & Porto Alegre & Albacete & Salford \\
\hline Población & 1.300 .000 & 153.636 & 216.103 \\
\hline Objetivo Principal & $\begin{array}{l}\text { Político } \\
\text { Social }\end{array}$ & $\begin{array}{l}\text { Político } \\
\text { Social }\end{array}$ & $\begin{array}{l}\text { Modernización } \\
\text { Social }\end{array}$ \\
\hline $\begin{array}{l}\text { Actores impulsores del pro- } \\
\text { ceso }\end{array}$ & $\begin{array}{l}\text { Confluencia entre un movimien- } \\
\text { to desde abajo y uno desde } \\
\text { arriba. }\end{array}$ & $\begin{array}{l}\text { Confluencia entre un movimiento } \\
\text { desde abajo y uno desde arriba. }\end{array}$ & Desde abajo. \\
\hline Proporción del presupuesto & $\begin{array}{l}\text { Fundamentalmente sobre inver- } \\
\text { siones, aunque se discute } \\
\text { sobre todo el gasto. }\end{array}$ & $\begin{array}{l}\text { Todo el presupuesto de gasto, pero sin } \\
\text { mucha concreción. También las } \\
\text { tasas y los precios públicos. }\end{array}$ & $\begin{array}{l}\text { Todo el presupuesto de gasto, aunque } \\
\text { aún está en proceso. }\end{array}$ \\
\hline Ámbito de aplicación & Zona, temática y ciudad & Ciudad & Ciudad \\
\hline Actores principales & Ciudadanos individuales & Asociaciones & Ciudadanos individuales \\
\hline $\begin{array}{l}\text { Partido Político que impulsa } \\
\text { la experiencia }\end{array}$ & Partido de los Trabajadores & Partido Socialista Obrero Español & Partido Laborista \\
\hline Fundaciones y ONG's & Sí & No & Sí \\
\hline
\end{tabular}

Fuente: Elaboración propia.

En primer lugar, en lo que respecta al tamaño de población vemos que Porto Alegre es la de mayor tamaño, aunque hay que tener en cuenta que Salford, como mencionamos con anterioridad, pertenece al área metropolitana conocida como Greater Manchester (que comprende, además de Manchester, los municipios de Salford, Bolton, Bury, Oldham, Rochdale, Stockport, Tameside, Trafford y Wigan, sumando entre todas una población de 2.482.328 habitantes).

En cuanto a los objetivos, aunque existen varios en todas las experiencias, lo cierto es que en cada una de ellas hay uno que prima sobre los demás. Porto Alegre buscaba aplicar una nueva forma de hacer política, distinta del neoliberalismo imperante en su país y que pensaban que mejoraría, mediante políticas redistributivas, la situación de los más desfavorecidos. En el caso de Salford, lo que se pretende es mejorar la calidad de los servicios y cambiar la imagen de la ciudad. Finalmente, en Albacete se persigue acercar la política a los ciudadanos, intentando que éstos sean partícipes de la gestión de su ciudad. 
Sobre la siguiente cuestión, actores que han sido los impulsores del proceso, habría que hacer alguna matización. Todas las experiencias surgen porque el alcalde y, en algunos casos, su equipo de gobierno creen que la participación de los ciudadanos es positiva para el desarrollo de la ciudad. Si los representantes políticos no son capaces de compartir alguna parcela de su poder con los ciudadanos no es posible llevar a cabo una experiencia de este tipo. No obstante, en algunos casos -como Porto Alegre y Albacete- la experiencia se desarrolla porque hay un encuentro entre las ideas participativas del responsable o responsables políticos y las de los movimientos sociales de la ciudad. Salford es, en cambio, fruto de una operación llevada a cabo «desde abajo» por algunas organizaciones sociales que entraron en contacto directo -a través de la cooperación internacional- con las prácticas brasileñas de democratización local. Como comentamos anteriormente, la ONG Community Pride, fundada en abril de 1999 con el apoyo de algunas organizaciones eclesiásticas y, más tarde, de Oxfam, efectuó algunos intercambios con las ciudades brasileñas de Porto Alegre y Recife. A continuación, en octubre de 2000, publicó un documento titulado «A Citizens Budget» («Un presupuesto ciudadano»), destinado a los administradores de Manchester y Salford. Posteriormente, organizó seminarios de formación para las autoridades locales de las dos ciudades y para los actores interesados procedentes de la sociedad civil. Estableció además un observatorio de investigación sobre el presupuesto público.

Respecto a la proporción del presupuesto priorizada por los presupuestos, en los tres casos teóricamente se está hablando de todo el presupuesto, aunque en la práctica existen diferencias importantes entre ellos. Una de las más notables, y que se ha estudiado poco, es el modelo racional de presupuesto utilizado en cada una de las ciudades, cuestión que tiene mucha importancia a la hora de permitir la participación de los ciudadanos.

También existen diferencias en el ámbito de aplicación. Porto Alegre comenzó su experiencia territorialmente (dividiendo la ciudad en dieciséis zonas o distritos), ampliando con el tiempo la participación a áreas temáticas y por último el conjunto de la ciudad. Por el contrario Albacete ha seguido el camino contrario: primero han sido los foros de ciudad, más adelante los foros temáticos (aunque sin regularidad) y ahora se están planteando los foros territoriales (de distrito o barrio). Salford siempre ha funcionado a nivel de toda la ciudad.

En las tres experiencias, los partidos políticos que las han puesto en marcha son partidos de izquierda, confirmando los resultados de la investigación de Sintomer (2004). En ella se señalaba que son los partidos de izquierda los que están más interesados en la temática: los comunistas y los verdes desde el principio y los socialistas más recientemente.

El papel de las ONG y las fundaciones ha sido muy importante en Salford; como ya hemos visto, también lo ha sido y lo es en Porto Alegre ${ }^{38}$. En España, sin embargo, las fundaciones y las ONG no juegan un papel importante en la difusión de la idea de presupuesto participativo, en la elaboración de las metodologías necesarias o en el asesoramiento técnico del proceso. Ello debería ser un motivo de reflexión.

\section{Conclusiones}

La participación de los ciudadanos en el presupuesto municipal puede llegar a convertirse en «el lugar» donde intentar reconstruir -con el tiempo y de manera colectiva- el concepto de «bien común», transformando las tensiones sociales en un «proyecto compartido» enmarcado en espacios autogestionados por la sociedad civil

${ }^{38} \mathrm{La}$ ONG Cidade ha tenido un papel muy importante en el desarrollo, durante todos estos años, del presupuesto participativo de Porto Alegre. Este papel puede ser aún más importante en estos momentos de cambio. 
-y marcados por un diálogo interactivo con las instituciones correspondientes-. Entre sus objetivos (de manera independiente o mediante asociaciones) caben destacar los siguientes: el desarrollo ético de las instituciones; el aumento del sentido cívico de los habitantes y de su capacidad para interpretar adecuadamente la complejidad del «derecho a la ciudad» para todos aquellos que viven en ella; y la difusión de formas de «solidaridad negociada», que permitan la redistribución justa de los recursos públicos a favor de las categorías con mayores desventajas culturales, sociales y económicas.

En cuanto a las experiencias estudiadas, es necesario señalar que, a pesar de no tener demasiados rasgos comunes y adolecer de falta de uniformidad, son importantes realmente por su diversidad, su flexibilidad y su adaptación rápida a los contextos locales. Estas experiencias no existen en forma aislada. Forman parte, antes que nada, de una cultura popular de participación y de relaciones entre el gobierno local y la sociedad. Suponen como precondición una ciudadanía movilizada y una clara voluntad política del alcalde y de las autoridades municipales.

Es necesario también resaltar la importancia del método sugerido por las matrices de distribución de los recursos ${ }^{39}$ elaborado por Community Pride, que adquiere un papel importante como instrumento técnico innovador para garantizar la transparencia en la toma de decisiones. Desde ese punto de vista, persigue acabar con uno de los puntos débiles de muchas experiencias europeas, especialmente de las españolas: la escasa atención prestada a las reformas administrativas que deberían acompañar a la ejecución del presupuesto participativo, con el fin de que la modernización de la maquinaria pública constituya un multiplicador de los efectos obtenidos. En la mayoría de los casos, sucede todo lo contrario.

No obstante, aunque este aspecto es importante, en estas experiencias no se trata solamente de introducir metodologías de gestión que faciliten la implicación del aparato administrativo del municipio en las etapas del ciclo participativo, sino sobre todo de introducir cambios fundamentales en la construcción de una cultura democrática.

Es importante señalar, como hemos podido ver en las experiencias estudiadas, que no existe una receta universal para iniciar una experiencia de participación presupuestaria. Ésta tiene que adecuarse a la situación y características locales, aunque existen ciertas condiciones que lo facilitan. De todas formas, aunque no llegaran a consolidarse las experiencias de presupuesto participativo en marcha, el hecho de plantearse otra forma de elaborar el presupuesto municipal en el que tengan participación directa los ciudadanos abre un espacio que no podrá cerrarse con facilidad.

\section{REFERENCIAS BIBLIOGRÁFICAS Y DOCUMENTALES}

Abers, R. (1998), «Learning Democratic Practice: Distributing Government Resources Through Popular Participation in Porto Alegre, Brazill, en M. Douglas y J. Friedmann, eds., Cities for Citizens. Chichester: John Wiley \& Sons.

Allegretti, G. y Herzberg, C. (2004), «El "retorno de las carabelas". Los presupuestos participativos de América Latina en el contexto europeo», Transnacional Institute and the Centre for Democratic Policy Making. Briefing Series, número 2004/5. Amsterdam y Madrid: Fundación de Investigaciones Marxistas.

Arenilla, M. (2004), La reforma administrativa desde el cindadano. Madrid: INAP.

Avilés, J. (2004), «Técnicas de organización de los Presupuestos Participativos», Temas para el Debate, 113: 45-49.

${ }^{39}$ El método elabora una media (calculada a través de pesos e indicadores) entre las necesidades expresadas por los ciudadanos participantes y las necesidades objetivas de la zona. 
Avritzer, L. (1999), «Public Deliberation at the Local Level: Participatory Budgeting in Brazil», en: http://www.ssc.wisc. edu/ wright/avritzer.pdf (consulta: 1 octubre 2005).

Avritzer, L. y Navarro, Z. eds. (2003), A inovação democrática: O orçamento participativo no Brasil. Sao Paulo: Cortes Editora.

Bacqué, M. H.; Rey, H. y Sintomer, Y. coords. (2004), Gestion de proximité et démocratie participative: les nowveaux paradigmes de l'action publique? París: La Découverte.

Baierle, S. (2003), «La experiencia brasileña en Presupuesto Participativo», Documento Base del Seminario de Lanzamiento de la Red Urb-al, 9, Porto Alegre.

Ballart, X y Ramió, C. (2000), Ciencia de la Administración. Valencia: Tirant lo Blanch.

Barceló, S. y Pimentel, Z. (2002), Radicalizar la democracia. Porto Alegre: un modelo de municipio participativo. Madrid: La Catarata.

Blanco, I. (2003), «Presupuestos Participativos en Rubí: innovación, límites y retos de una experiencia pionera», Comunicación presentada en Jornadas sobre participación ciudadana «Tú decides». Ayuntamiento de Jerez de la Frontera, 29-31 de octubre.

Cabannes, Y. (2003a), «Introducción y Metodología», Documento Base del Seminario de Lanzamiento de la Red Urb-al número 9 , Porto Alegre.

- (2003b), «Presupuestos Participativos en ciudades europeas y latinoamericanas: lecciones aprendidas de las experiencias», Documento Base del Seminario de Lanzamiento de la Red Urb-al número 9, Porto Alegre.

Chandler, J. A. ed. (2000), Comparative Public Administration. Routledge: Londres.

CIDADE (2005), Boletim CIDADE, 14, año V, abril-mayo.

Community Pride Initiative (2003), «Building a People's Budget. Report of the Salford budget matrix study», en: http://www.participatorybudgeting.org.uk (consulta: 1 agosto 2005).

- (2005a), «Breathing life into democracy. The power of participatory budgeting», <http://www.participatorybudgeting.org.uk> (consulta: 1 agosto 2005).

- (2005b), «Margins to mainstream», documento de trabajo, Salford.

Fedozzi, L. (1997), Orçamento Participativo-Reflexões sobre a Experiência de Porto Alegre. Porto Alegre: Tomo.

- (2000), O poder da Aldeia: gènese e història do Orçamento Participativo de Porto Alegre. Porto Alegre: Tomo.

Genro, T. (1997), «El presupuesto participativo y la democracia», en: http://www.arrakis.es/ ldrain/demopunk/ sp/direct/porto/porto1.html (consulta: 15 julio 2005).

- (2000), Reinventar el futuro. Democracia y socialismo en la era de la globalización. Barcelona: Del Serval.

Gret, M., y Sintomer, Y. (2003), Porto Alegre. La esperanza de otra democracia. Barcelona: Debate.

Marquetti, A. (2000), «Participatory budgeting in Porto Alegre», Indicator S. A., 17 (4): 71-78.

- (2003), «Participação e Redistribuição: o Orçamento Participativo em Porto Alegre», en L. Avritzer, y Z. Navarro, eds., A inovação democrática: O orçamento participativo no Brasil. Sao Paulo: Cortes Editora.

Navarro, Z. (2000), «Inventando o futuro das cidades: pequena história do Orçamento Participativo em Porto Alegre», en H. Rattner, org., Brasil no limiar do Século XXI, São Paulo: Edusp.

- (2002), «O’Orçamento Participativo» de Porto Alegre (1989-2002): um conciso comentário crítico». en: http://www.democraciaparticipativa.org (consulta: 15 julio 2005).

Olmeda, J. A. y Parrado, S. (2000), Ciencia de la Administración. Los Sistemas Administrativos. Madrid: UNED.

Parrado, S. (2002), Sistemas administrativos comparados. Madrid: Tecnos.

Pérez Castell, M. (2004), «La experiencia de los Presupuestos Participativos en Albacete», Temas para el Debate, 113: 54-57. 
Pineda, C. (2002a), «Mejora de la gobernabilidad en el nivel de Gobierno local: participación de más actores en el proceso presupuestario». Ponencia presentada en el VI Congreso del CLAD, Lisboa, 8-11 octubre.

- (2002b), «Gobiernos locales: participación ciudadana en el proceso presupuestario», Gestión y Análisis de Politicas Públicas, 22: $161-173$

- (2003), «La participación ciudadana en el proceso presupuestario dentro del ámbito local: consideraciones generales y la experiencia de Albacete». Ponencia presentada en el VI Congreso de Ciencia Política y de la Administración, Barcelona, septiembre.

- (2004a), «Posibilidades y condicionamientos de los Presupuestos Participativos», Temas para el Debate, 113: 41-45.

- (2004b), «Los Presupuestos Participativos desde una perspectiva comparada». Ponencia presentada en el VIII Congreso de Sociología, Alicante, septiembre.

- (2004c), «Los Presupuestos Participativos en España: un balance provisional», Revista de Estudios Locales, 78: 64-76.

Pineda, C. y Sanz, A. (1999), «Presupuesto y participación: la experiencia de Porto Alegre y Kerala». Ponencia presentada en el IV Congreso de Ciencia Politica y de la Administración. Granada, septiembre-octubre.

Pires, V. (2000a), «Límites y potencialidades del presupuesto participativo», Revista Internacional de Presupuesto Público (ASIP), 42. en: http://www.asip.org.ar/es/revistas/ (consulta: 30 julio 2005).

- (2000b), «Participación de la sociedad en los procesos presupuestarios: la experiencia brasileña reciente». Ponencia presentada en el XXVII Seminario Internacional de Presupuesto Público. Madrid, noviembre.

Rodríguez Álvarez, J. M. (2004), La Ley de Medidas para la Modernización del Gobierno local. Barcelona: Bayer Hons.

Rodríguez Prieto, R. (2005), Ciudadanos soberanos. Participación y democracia directa. Madrid: Almuzara.

Sousa Santos, B. de (1998), «Participatory budgeting in Porto Alegre: towards a redistributive justice», Politics and Society, 26 (4): 416-510.

- (2002), «Orçamento Participativo em Porto Alegre: para uma democracia redistributiva», en B. de Sousa Santos, org., Democratizar a democracia. Os caminhos da democracia participativa. Rio de Janeiro: Civilização Brasileira.

Sintomer, Y. (2004), «Los presupuestos participativos en Europa: retos y desafíos». Comunicación presentada en el IX Congreso Internacional del CLAD sobre la Reforma del Estado y de la Administración Pública. Madrid, noviembre.

- (2005), «La participación ciudadana como tendencia política europea», Seminario Politicas Sectoriales y participación ciudadana, Ayuntamiento de Madrid. Madrid, 25 de octubre.

De Souza, U. (2001), Orcamento participativo: a experiencia de Rio Grande do Sul. Montevideo: Instituto de Cooperación y Desarrollo.

Utzig, J. E. (1999), «Orçamento Participativo e Performance Governamental», paper escrito para el programa SPURS (Special Program for Urban and Regional Studies). Cambridge, Mass.: MIT, primer semestre 1999.

- (2000), «El presupuesto participativo en Porto Alegre: análisis basado en el principio de legitimidad democrática y el criterio de desempeño gubernamental», documento publicado por el Banco Mundial.

Villasante, T. (2004), Los desbordes de técnicos y vecinos en los movimientos ciudadanos actuales. Barcelona: El Viejo Topo.

Wainwright, H. (2000), «Streets Ahead», The Guardian, 21 de junio del 2000.

Wampler, B. (2002), «Orçamento participativo: uma explicação para as amplas variações nos resultados», en L. Avritzer y Z. Navarro, eds., A inovação democrática: O orçamento participativo no Brasil. Sao Paulo: Cortes Editora.

Ziller, J. (2005), Administrations Comparées. Les systèmes politico-administratifs de l'Europe des douze. París: Montchrestien. 